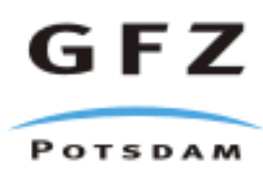

Originally published as:

Schmid, R., Rothacher, M., Thaller, D., Steigenberger, P. (2005): Absolute phase center corrections of satellite and receiver antennas. Impact on GPS solutions and estimation of azimuthal phase center variations of the satellite antenna. - GPS Solutions, 9, 4, 283-293,

DOI: 10.1007/s10291-005-0134-X. 


\section{Absolute phase center corrections of satellite and receiver antennas}

Impact on global GPS solutions and estimation of azimuthal phase center

variations of the satellite antenna

Ralf Schmid, Markus Rothacher, Daniela Thaller, Peter Steigenberger

R. Schmid

Institut für Astronomische und Physikalische Geodäsie,

Technische Universität München,

80290 München,

Germany

schmid@bv.tum.de

+49-89-289-23193

+49-89-289-23178

M. Rothacher, D. Thaller, P. Steigenberger

Forschungseinrichtung Satellitengeodäsie,

Technische Universität München,

80290 München,

Germany

rothacher@bv.tum.de

daniela.thaller@bv.tum.de

steigenberger@bv.tum.de

Keywords: Satellite Antenna, Phase Center Variations, Tropospheric Zenith Delay, Elevation CutOff

Results of the estimation of azimuth-dependent phase center variations (PCVs) of GPS satellite antennas using global GPS data are presented. Significant variations of up to $\pm 3-4 \mathrm{~mm}$ are demonstrated that show excellent repeatability even over years. The application of the azimuthal PCVs besides the nadir-dependent ones will lead to a further reduction of systematic antenna effects. In addition, the paper focuses on the benefit from a possible transition from relative to absolute PCVs. Apart from systematic changes in the global station coordinates, one can expect the GPS results to be less dependent on the elevation cut-off angle. This, together with the significant reduction of tropospheric zenith delay biases between GPS and VLBI, stands for an important step towards more consistency between different space geodetic techniques.

\section{Introduction}

For several years, absolute receiver antenna phase center variations (PCVs) from two independent approaches (calibration in an anechoic chamber and field calibration with a robot) have been 
available that are in good agreement among each other as well as with the relative PCVs published by the IGS (International GPS Service; see e.g. Rothacher 2001). Nevertheless still the latter are used, although it is well known that systematic errors are the consequence (e.g. Schmid et al. 2005). The problem of a terrestrial scale change of about 15 ppb w.r.t. ITRF2000 that initially prohibited the adoption of absolute PCVs could be solved in the meantime by the estimation of nadir-dependent satellite antenna PCVs (Schmid and Rothacher 2003). The latter are also called "absolute”, namely in terms of compatibility to the absolute receiver antenna corrections and not in a real absolute sense. At the moment, several IGS Analysis Centers are working on the generation of best possible satellite antenna correction values in order to prepare for a transition from relative to absolute PCVs within the IGS.

Ge and Gendt (2005) showed that it is not sufficient to use block-specific antenna correction values, as there are significant differences in the phase center behavior between certain subgroups of the satellite blocks or even between individual satellites (see also Schmid et al. 2005). This holds particularly true for the phase center offsets, but possibly for the PCVs as well. First results of the reprocessing of a global GPS network performed at the Technical Universities of Munich and Dresden (Steigenberger et al. 2004) pointed at the necessity to use long periods of data for the estimation of phase center offsets and variations of individual satellites, as weeks with poor determinability alternate with periods of high accuracy for the estimated parameters (high accuracy for small angles between the orbit plane and the direction to the sun). Since these long term results are not yet fully available, the present paper will not consider the PCV details of individual satellites.

Rather, the focus of this paper is on a more detailed description of the overall PCV model of the satellite antenna as well as on the benefit from the transition from relative to absolute receiver and satellite antenna PCVs, when estimating global GPS parameters. The first paragraph of the results section addresses the completion of the nadir-dependent satellite antenna PCV models by estimating antenna correction values for Block I satellites by means of data from 1994. The second paragraph is devoted to azimuth-dependent satellite antenna PCVs that may be expected due to the antenna assembly and the power supply (Czopek and Shollenberger 1993). The estimates are primarily analyzed in terms of repeatability and resolution. Recently, PCV maps including azimuthal variations were also derived from on-orbit data of Jason-1 and GRACE A and B by Haines et al. (2004).

Finally, the third paragraph of the results section deals with the comparison of global GPS solutions estimated using relative and absolute PCVs, respectively, for the receiver and the satellite antennas. At first, coordinate sets corresponding to different elevation cut-off angles are compared in order to confirm the suggestion that the use of absolute PCVs might reduce the dependence of coordinate results on the elevation cut-off angle (Schmid and Rothacher 2003). The fact that the mismodeling of an elevation-dependent effect can cause coordinate results depending on the selected elevation cut-off angle was stated by Elósegui et al. (1995), Hatanaka et al. (2001) and 
others. At the end of the paper, the influence of antenna PCVs on the biases between total tropospheric zenith delays derived from different space geodetic techniques is discussed. Schuh and Böhm (2003) who analyzed the GPS and VLBI (very long baseline interferometry) derived troposphere parameters for identical times at eleven co-located sites found out that all mean values of the total zenith delays derived by GPS are larger than those derived by VLBI. Haas et al. (2003) get similar results for the Onsala Space Observatory from an analysis of long time series of integrated precipitable water vapor (IPWV) from four different techniques (GPS, VLBI, water vapor radiometers (WVR) and radiosondes). The reduction of systematic biases between GPS, VLBI and other space geodetic techniques such as SLR (satellite laser ranging) are of particular importance with regard to the combination of the different techniques and the establishment of an Integrated Global Geodetic Observing System (IGGOS; Rummel et al. 2000, Rummel et al. 2002). Any unmodeled systematic effect present within one single technique will complicate or even rule out such a combination.

Let us start, however, by describing the relation between "horizontal” satellite antenna offsets and azimuthal PCVs, the antenna assembly of the different satellite blocks, and the data sets used.

\section{Basic relations}

As a first step, we have to define the azimuth angle under which a specific station is seen from the satellite. We adopt the convention here that the y-axis corresponds to the nominal rotation axis of the solar panels, the z-axis points towards the Earth, and the x-axis completes the right-hand system (Hugentobler et al. 2001). In analogy to the receiver antenna where the azimuth counts clockwise from the north towards the east direction, the azimuth at the satellite antenna is also chosen to count clockwise for these studies, but now from the y-axis in the satellite-fixed coordinate system towards the $\mathrm{x}$-axis when looking towards the z-axis.

In the case of both, receiver and satellite antennas, PCVs are always correlated with the phase center offset. A satellite antenna offset $\Delta z$ in z-direction causes nadir-dependent PCVs (Schmid and Rothacher 2003), whereas "horizontal” offsets show up as azimuthal variations. If the phase center is shifted by ( $\Delta x, \Delta y$ ) from its original position (see Fig. 1), an observation in the azimuth direction $\alpha$ is affected by

$\Delta x y(\alpha)=\sqrt{\Delta x^{2}+\Delta y^{2}} \cdot \cos \left(\alpha_{\Delta}-\alpha\right)$

where $\alpha_{\Delta}$ is the azimuth angle of the shifted phase center as seen from the original phase center.

That means that a maximum effect can be seen in the direction $\alpha_{\Delta}$ of the offset, whereas perpendicular directions are not affected. In addition, Figure 2 gives the relationship concerning the nadir angle $z^{\prime}$ : no effect in nadir direction, maximum effect for $z_{\max }^{\prime} \approx 14.3^{\circ}$. For this reason, an error in the modeling of the horizontal offset shows up in the satellite antenna PCVs $\phi^{\prime}$ 
as a cosine curve in azimuth direction $\alpha$ with increasing amplitude for increasing nadir angles $Z^{\prime}$ :

$\Delta \phi^{\prime}\left(z^{\prime}, \alpha\right)=\sqrt{\Delta x^{2}+\Delta y^{2}} \cdot \sin z^{\prime} \cdot \cos \left(\arctan \frac{\Delta x}{\Delta y}-\alpha\right)$

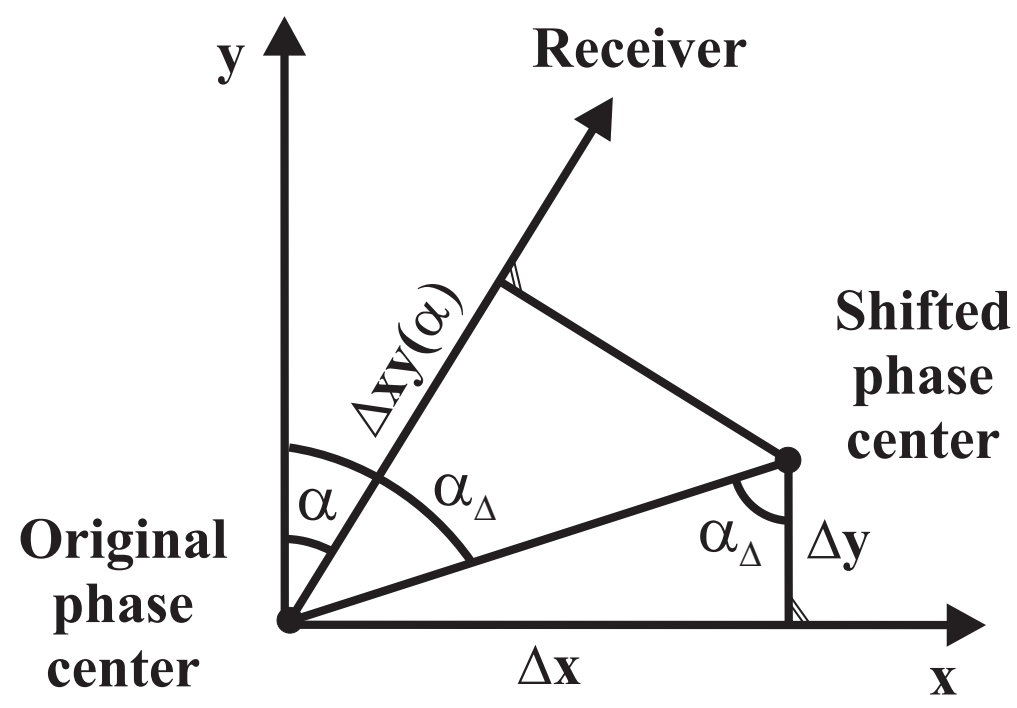

Fig. 1

Relationship between horizontal offset and azimuth direction

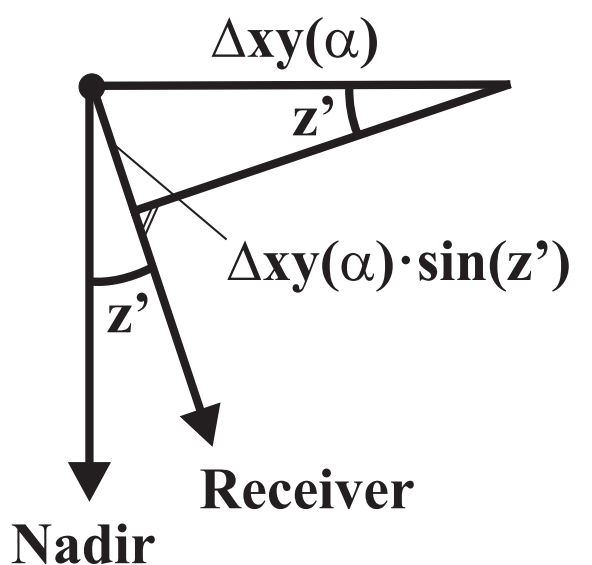

Fig. 2

Relationship between horizontal offset and nadir angle

In order to get the size of the horizontal offset error from estimated azimuthal PCVs $\Delta \phi^{\prime}\left(z_{0}^{\prime}, \alpha\right)$ with $z_{0}^{\prime}>0$, the latter can be fit to the cosine function

$f(\alpha)=A \cdot \cos \left(\alpha_{\Delta}-\alpha\right)$ 
with the amplitude $A$ and the phase shift $\alpha_{\Delta}$. The horizontal offsets to be compensated for with reversed sign are then given by

$$
\begin{aligned}
& \Delta x=\frac{A}{\sin z_{0}^{\prime}} \cdot \sin \alpha_{\Delta} \text { and } \\
& \Delta y=\frac{A}{\sin z_{0}^{\prime}} \cdot \cos \alpha_{\Delta}
\end{aligned}
$$

As regards the antenna assembly of the different satellite blocks (Block I, Block II/IIA, Block IIR), not all the facts of interest in connection with azimuthal PCVs are clearly known. According to Aparicio et al. (1995), each antenna comprises twelve helical elements, arranged in two concentric circles on the Earth-facing satellite panel. The inner circle is composed of four elements fed with $90 \%$ of the total power, and the outer circle contains the eight remaining elements. Even though the radii of the two circles vary from block to block, there is no indication of an unequal spacing of the single elements within one circle in any of the publications. Czopek and Shollenberger (1993) have shown that the field intensity is not completely symmetrical in the plane perpendicular to the z-axis, because there are only four elements in the center. The maximum gain is achieved in the two planes that intersect two elements of the center quad. The variation of the field intensity caused by the outer circle is minimized due to the increase in the number of elements, providing greater symmetry. As a result of that and due to the fact that the outer circle is only fed with $10 \%$ of the total power, one may expect to see mainly a fourfold pattern in the azimuthal PCVs. This is also clearly demonstrated in Figure 3 of Haines et al. (2004).

The orientation of the twelve elements with respect to the y-axis can only be obtained from drawings. According to Czopek and Shollenberger (1993), the y-axis points exactly in the direction between two elements of the inner circle for the Block I satellites, as can be seen in Figure 3. 


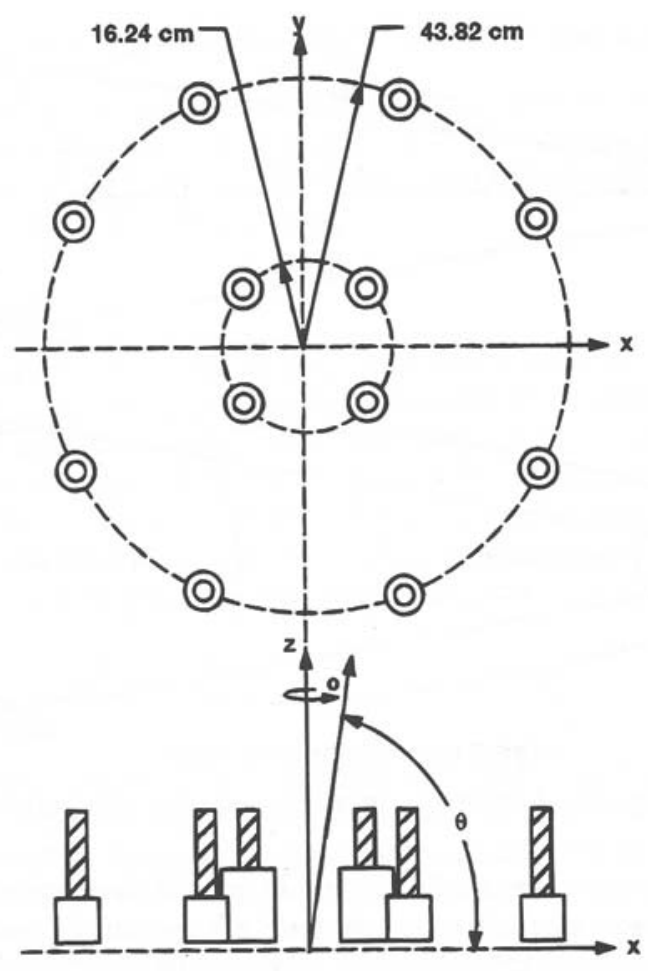

Fig. 3

Block I antenna assembly (Czopek and Shollenberger 1993)

Degnan and Pavlis (1994) describe the locations of a laser retroreflector array carried by two Block IIA satellites and of the antenna phase center with respect to the center of mass (see Fig. 4). The drawing also shows that the y-axis points towards an element of the outer circle, the four elements of the inner circle being located at azimuth $67.5^{\circ}, 157.5^{\circ}, 247.5^{\circ}$ and $337.5^{\circ}$. It has to be noted that the drawing is not drawn to scale, as the four elements of the inner circle are not tangent to each other in reality (cf. Fig. 1 in Mader and Czopek 2002). The orientation of the antenna elements mounted on the Block IIR satellites is unknown to the authors so far. 


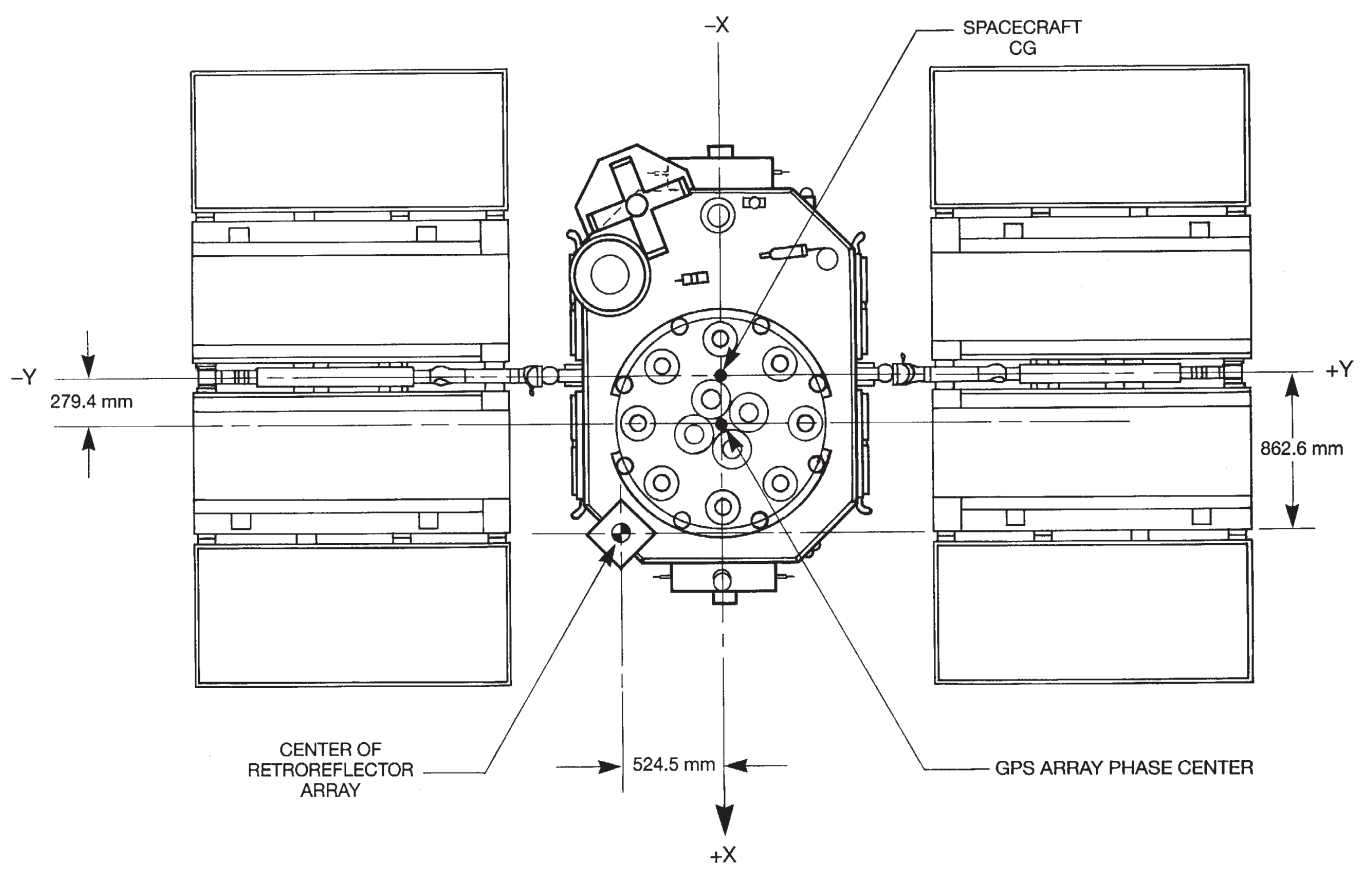

Fig. 4

Block II antenna assembly (Degnan and Pavlis 1994)

The PCVs were modeled as two-dimensional piece-wise linear functions with user-defined resolution in both elevation and azimuth direction. The value in nadir direction was constrained to be identical for all different azimuth directions, and the sum of all PCV values was constrained to be zero. As it was not possible to estimate separate phase center corrections for L1 and L2, all results refer to the ionosphere-free linear combination LC (cf. Schmid and Rothacher 2003).

\section{Input data}

The authors estimated one-day GPS solutions using double-difference phase data from globally distributed IGS stations with the Bernese GPS Software (Hugentobler et al. 2005) for three different time spans: January 1-9, 1994, July 14-19, 2002 and October 17-31, 2002. The data set from 1994 was generated as part of the reprocessing of a global GPS network performed by the Technical Universities of Munich and Dresden (Steigenberger et al. 2004). Due to the fact that three Block I satellites (PRN03, 12, 13) were active in early 1994, these data allow to estimate phase center correction values for that specific satellite block, too. Moreover, the data are interesting in terms of long-term consistency of the estimated parameters. The second set (July 2002) corresponds to the data used in (Schmid and Rothacher 2003). During the third time span the VLBI campaign CONT02 (see below) took place, so that the tropospheric estimates from GPS could be compared to independent VLBI results at co-located sites.

CONT02, a two-week campaign of continuous VLBI observations, was initiated by the International VLBI Service for Geodesy and Astrometry (IVS). The data of the eight participating 
stations were analyzed at Deutsches Geodätisches Forschungsinstitut (DGFI) with OCCAM (Titov et al. 2001). As great care was taken to use identical models and the same parameterization for the common parameters in the GPS and the VLBI analysis, a comparison of the tropospheric parameters derived from the two independent techniques should be meaningful (Thaller et al. 2005). For further comparisons a preliminary data set of one of the water vapor radiometers located at Onsala was available (Elgered and Haas 2003).

\section{Results}

The satellite antenna PCVs were estimated together with all relevant global parameters, namely site coordinates, site-specific troposphere parameters, orbit parameters and Earth rotation parameters. For the receiver antennas, absolute phase center corrections from robot calibrations were applied (Wübbena et al. 2000). For further details see (Schmid and Rothacher 2003). Due to an averaging over approximately 150 stations available, at least as regards the two data sets from 2002, local effects (i.e. effects not common to all stations) such as multipath or troposphere variations are expected to have only minor influence on the results.

\section{Nadir-dependent phase center variations}

The data set from 1994 was used to get phase center correction values for Block I. From the average phase pattern of nine days an offset correction in z-direction of $\Delta z_{I}=+1.149 \mathrm{~m}$ was derived, which results in an overall offset of $2.003 \mathrm{~m}$. The first value is of similar magnitude as those given in (Schmid and Rothacher 2003) for the other two satellite blocks:

$\Delta z_{\text {II /IIA }}=+1.315 \mathrm{~m}, \Delta z_{I I R}=+1.333 \mathrm{~m}$. This indicates that all satellite antenna offsets used by the IGS so far are quite consistent. When comparing the remaining phase pattern of Block I (estimated from the data from 1994) with those of the other blocks (data of 2002; see Fig. 5), it is obvious that the accuracy of the Block I PCVs is worse although more one-day solutions were processed. This is due to the small number of less than fifty stations operational in early 1994 and the small number of only three Block I satellites. One can see, however, that the nadir-dependent behavior of the Block I antenna differs significantly from that of Block II/IIA and Block IIR. 


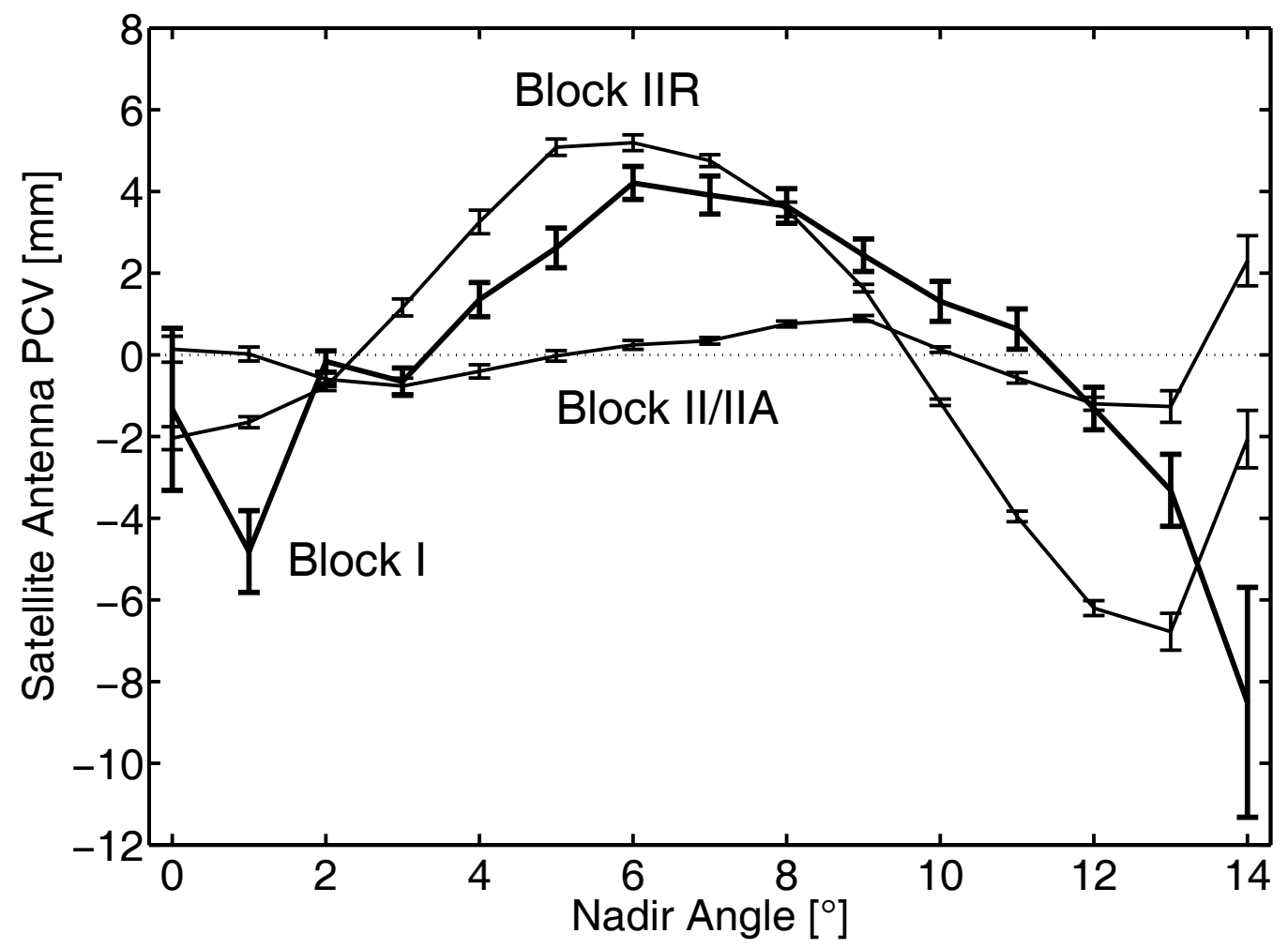

Fig. 5

Mean Block I phase pattern (January 1-9, 1994) compared to Block II/IIA and Block IIR (July 1419, 2002) together with the standard deviation derived from averaging over all daily solutions

Moreover, the data from 1994 could be used to check the consistency of the Block II/IIA pattern at two time intervals more than eight years apart. It has to be noted that differences of up to $3 \mathrm{~mm}$ show up when comparing the results from 1994 and 2002, ignoring the maximum nadir angle that is poorly determined. These discrepancies could arise from a scale drift of about 0.2 ppb per year that appears in the reprocessing results using absolute PCVs (Steigenberger et al. 2004). Another explanation could be the slow change in the composition (number of Block I, II/IIA, IIR satellites) of the space segment over time.

\section{Azimuth-dependent phase center variations}

Before discussing the actual results of the estimation of azimuthal PCVs, it is worth considering the distribution of observations with respect to the azimuth angle for one particular satellite. Figure 6 shows the mean percentage of the total of all observations falling into each interval of $10^{\circ}$ for PRN28. The mean percentage is calculated from six one-day solutions indicated by crosses (x) in the figure. As they are sometimes printed one upon the other, for most intervals less than six crosses are visible, however. According to Figure 6, the observations are not evenly distributed and this circumstance recurs every day. This means that some parts of the antenna are poorly covered by observations, making the determination of PCVs difficult in some cases. 


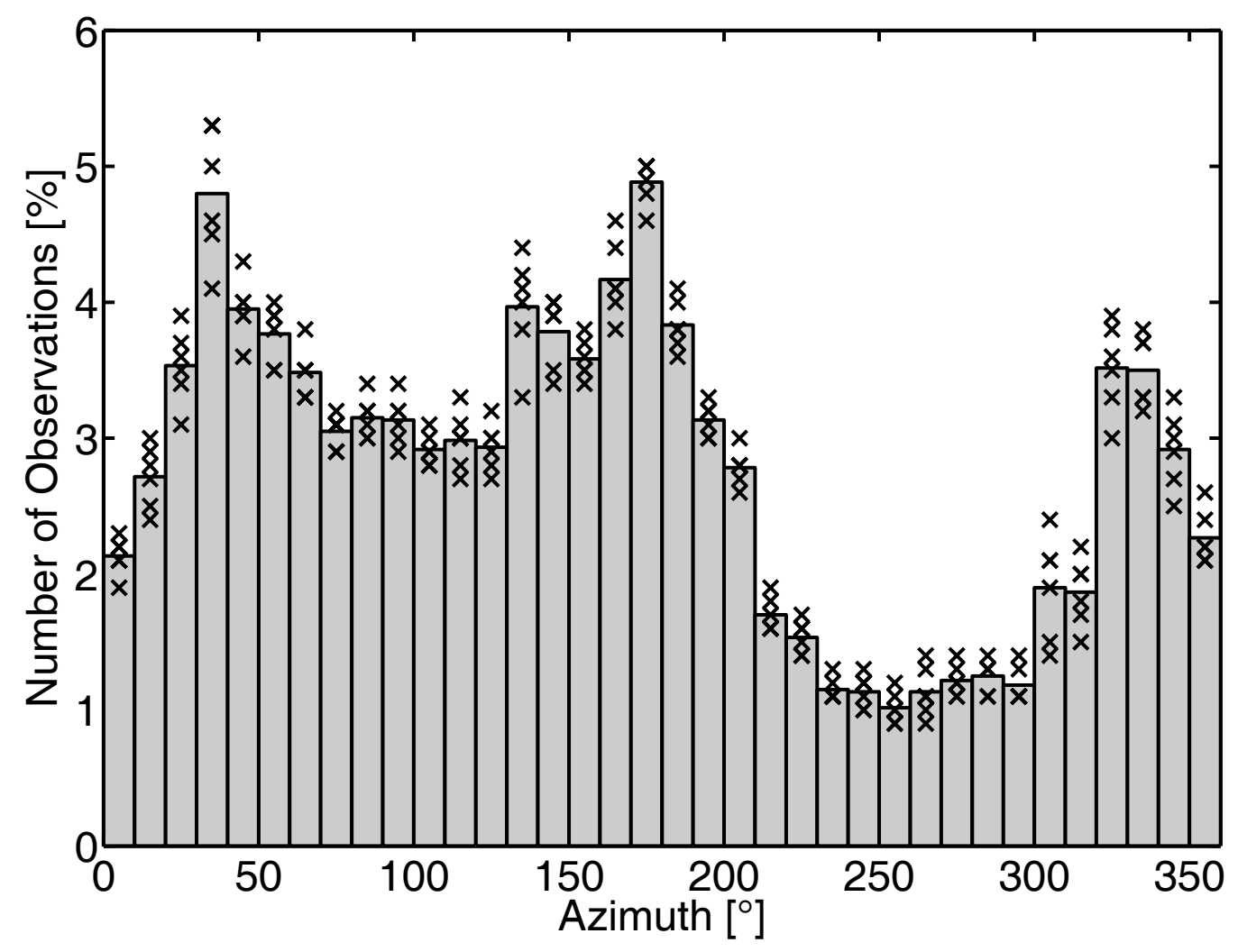

Fig. 6

Mean distribution of observations with respect to the azimuth angle for PRN28 (July 14-19, 2002). The crosses $(\mathrm{x})$ denote the distribution for the six individual days

Due to this observation distribution, the azimuthal PCVs were modeled as simply as possible for most of the following investigations (see Fig. 7): one linear function between the nadir point and the maximum nadir angle $\left(14^{\circ}\right)$ per azimuth direction was estimated. The resolution in azimuth direction was mostly chosen to be $10^{\circ}$. Following this strategy, one has to be aware of the distribution of observations with respect to the nadir angle given in (Schmid and Rothacher 2003). As more than $60 \%$ of the observations correspond to nadir angles above $10^{\circ}$, observations at low nadir angles do not have much influence on the slope of the straight line. In the figures to come, the circular curve at a nadir angle of $14^{\circ}$ shown in Figure 7 is plotted as a function of the azimuth angle. 


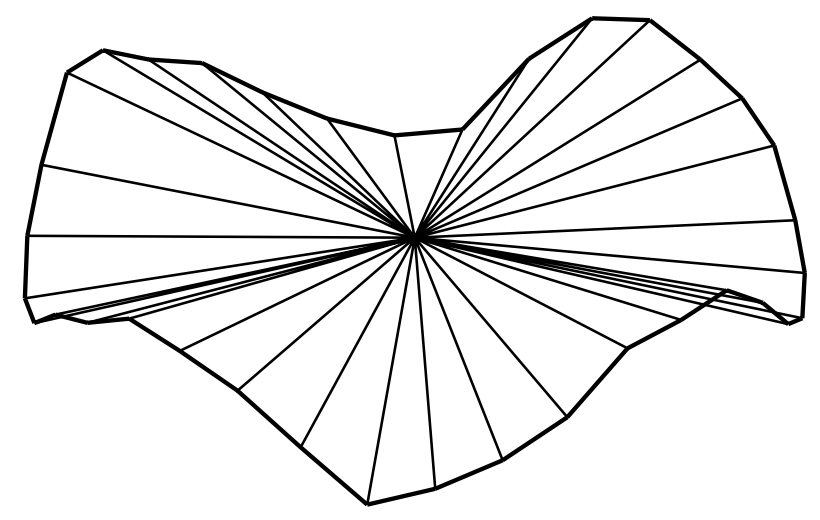

Fig. 7

Illustration of azimuthal PCVs modeled as linear functions: one value in nadir direction and one value at the maximum nadir angle for each azimuth direction

As described above, the azimuthal PCVs are always overlaid by the effect of phase center offset errors in the xy-plane. In order to eliminate this dominating and disturbing effect, one offset correction ( $\Delta x, \Delta y$ ) was applied to each one-day solution. Whereas the daily offset corrections are subject to strong variations (mainly due to correlations with orbital parameters) and necessitate long time spans of data for a precise determination, the azimuthal PCVs are much more stable.

In order to check the repeatability between different satellites carrying the same antenna, PCV estimates for single satellites of one satellite block were compared to a pattern estimated using the observations of all satellites of the block. Figure 8 shows the azimuthal variations averaged over six days for individual satellites of Block IIR and the block-specific mean pattern. First of all, one has to note that all the curves are in phase. This demonstrates that all Block IIR antennas are oriented consistently towards the y-axis in the satellite-fixed coordinate system. This also holds for Block II/IIA. In addition, the differences of individual patterns to the mean pattern are of major interest. Typical differences are of the order of $2 \mathrm{~mm}$. Larger deviations can mostly be explained by a lack of observations. As an example, the pattern of PRN28 that is missing in Figure 8 is off by about $3 \mathrm{~cm}$ between $220^{\circ}$ and $240^{\circ}$ azimuth, where only very few observations are available on all six days, as can be seen in Figure 6. 


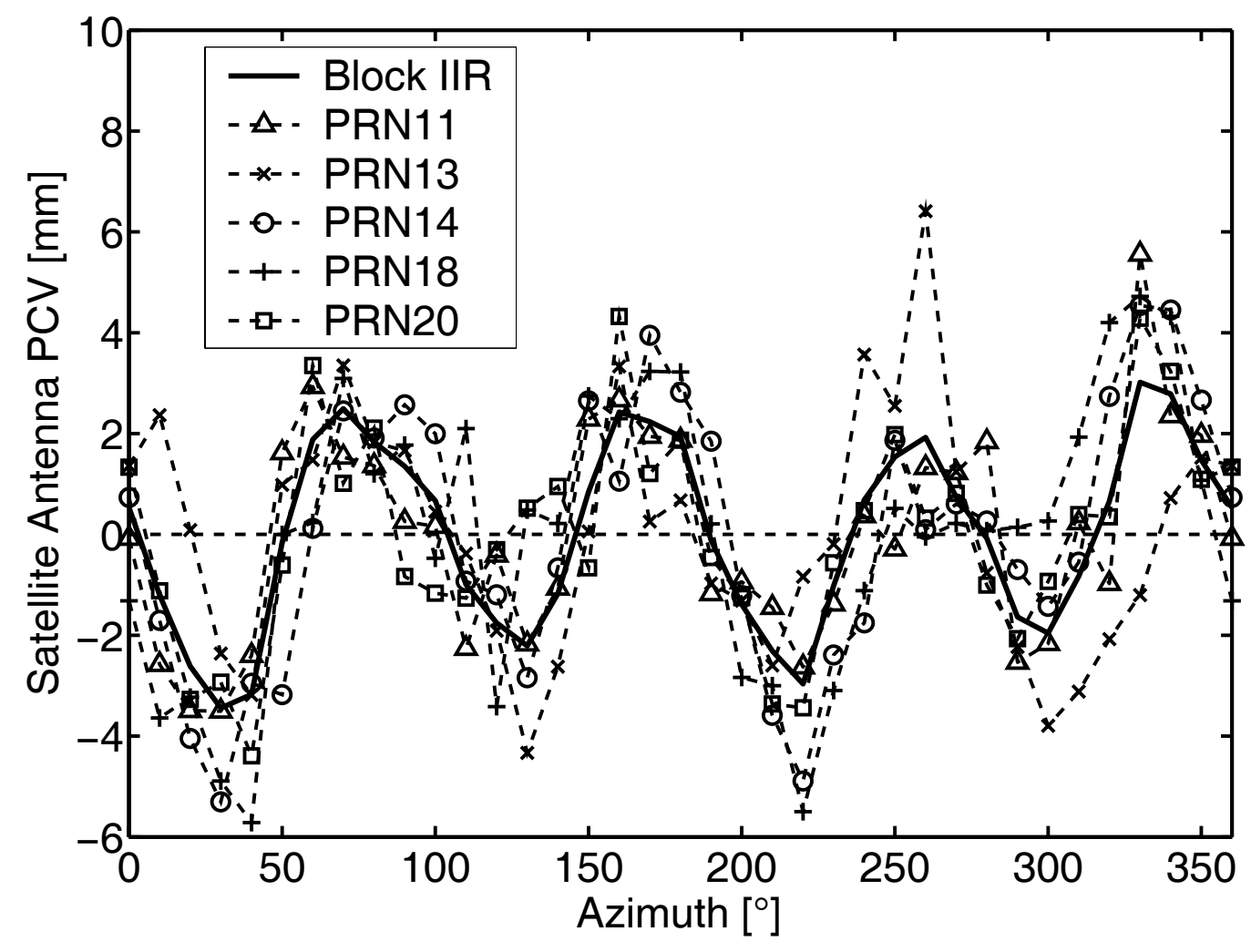

Fig. 8

Azimuthal PCVs at a nadir angle of $14^{\circ}$ for individual satellites of Block IIR (July 14-19, 2002)

An impression of the daily repeatability can be obtained by comparing the one-day estimates, as shown in Figure 9. Due to averaging over all satellites of the same block, the differences between the one-day estimates and the six-day mean are reduced to a maximum of $1 \mathrm{~mm}$ only. If the azimuthal resolution is decreased from $10^{\circ}$ to $5^{\circ}$, the signal gets noisier again, as one would expect. 
Azimuthal Resolution: $10^{\circ}$

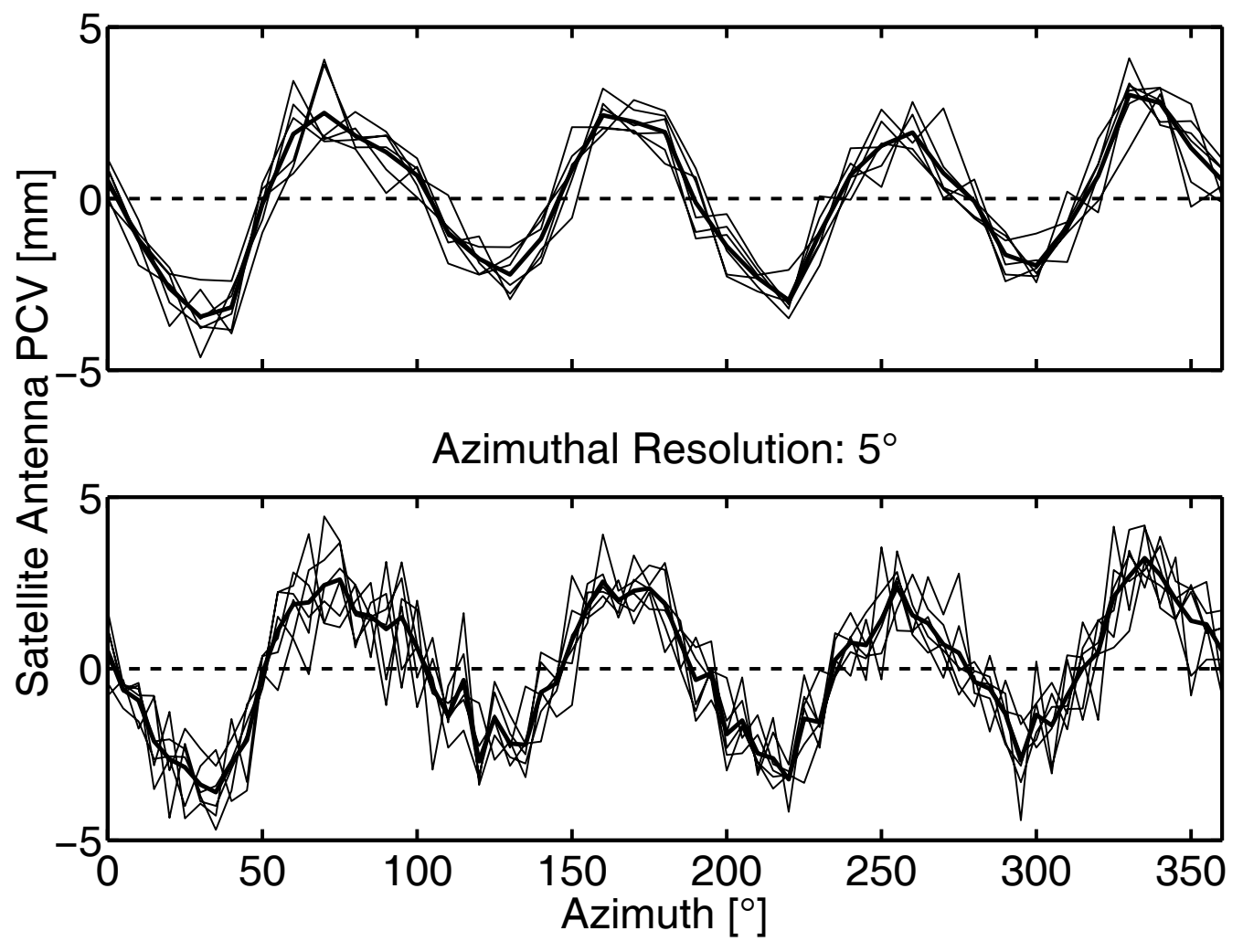

Fig. 9

Daily repeatability for the azimuthal PCVs of Block IIR for a nadir angle of $14^{\circ}$ and two different resolutions in azimuth (July 14-19, 2002)

Figure 10 shows the results of an unweighted averaging of the block-specific azimuthal PCVs over seven (1994) resp. six (2002) days for all satellite blocks. All three curves reach values of up to 3$4 \mathrm{~mm}$ for the given nadir angle of $14^{\circ}$. The error bars indicated in the figure give the standard deviation of the weekly mean (solely derived from the averaging). The fourfold pattern that was expected due to the reasons given above clearly shows up in the azimuthal variations of Block II/IIA and IIR. All amplitudes being of comparable magnitude, the PCVs of Block IIR seem to be fully symmetrical, whereas Block II/IIA shows a noticeable asymmetry. As regards Block I, a twofold pattern could be considered, if the peak between $80^{\circ}$ and $90^{\circ}$ azimuth was ignored. The accuracy of the Block I PCVs is worst, due to the reasons already mentioned above (only three satellites and less than fifty stations in 1994). Although the Block II/IIA PCVs from 1994 are also more poorly determined than those from 2002, it has to be pointed out that the agreement between the two curves is excellent. This suggests that the differences found earlier for the nadir-dependent PCVs (see section above) are not caused by possible changes of the antenna behavior, but rather by uncertainties due to correlations with other estimated parameters. 


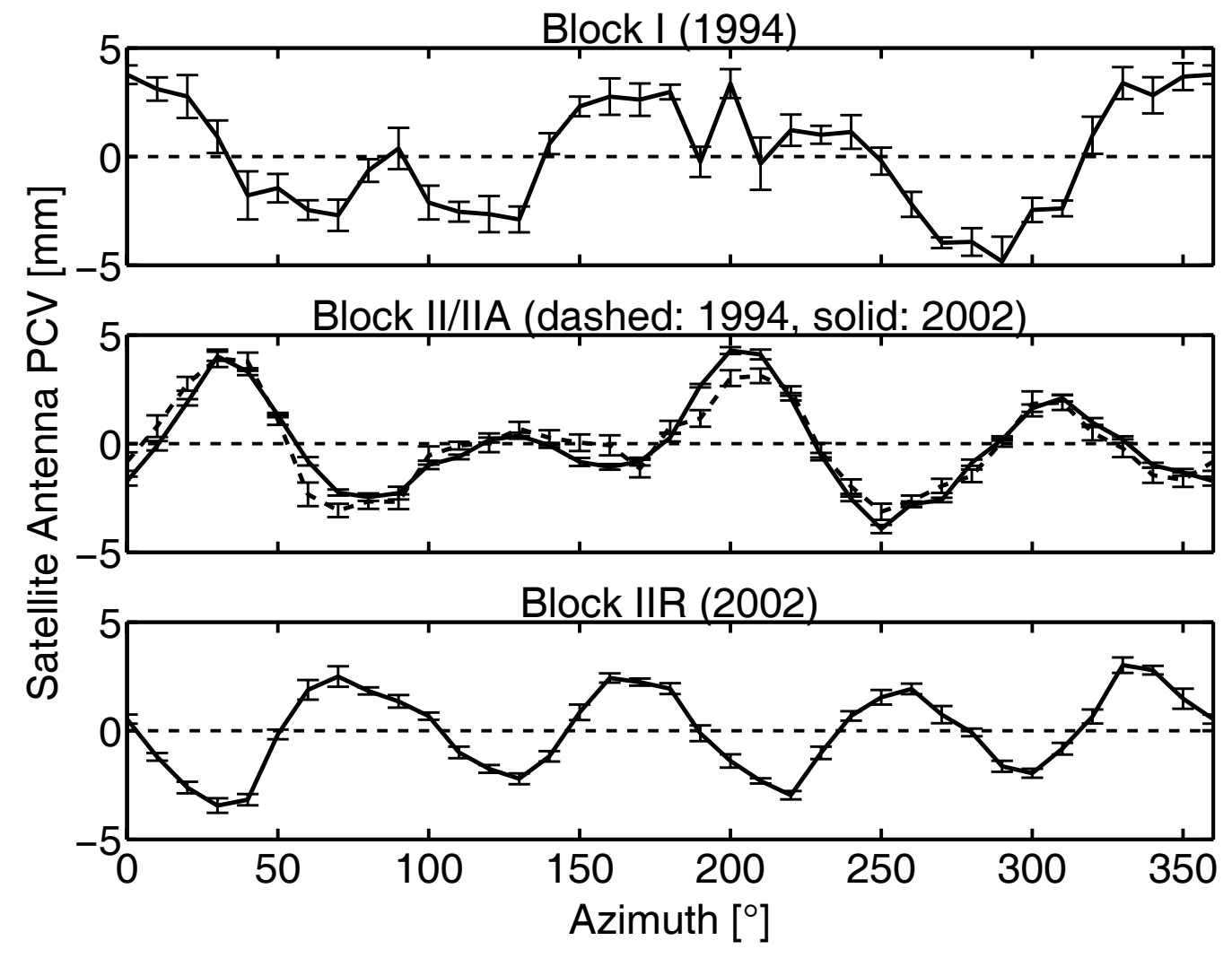

Fig. 10

Mean azimuthal satellite antenna PCVs with standard deviation for all three satellite blocks (January 1-7, 1994 resp. July 14-19, 2002)

As already shown (Fig. 9), a higher resolution in azimuth as well as in nadir direction increases the estimation noise. But whereas a resolution of $10^{\circ}$ in azimuth direction seems to be sufficient for the representation of the PCVs, the resolution in nadir direction used above $\left(14^{\circ}\right)$ is certainly not. In order to demonstrate that, the nadir angle resolution for Block II/IIA was set to $5^{\circ}$ in Figure 11 . It is interesting to see that the curve corresponding to a nadir angle of $5^{\circ}$ only exhibits two maxima, whereas the other two curves show four maxima. The phenomenon of a twofold pattern is already known from Block I. 


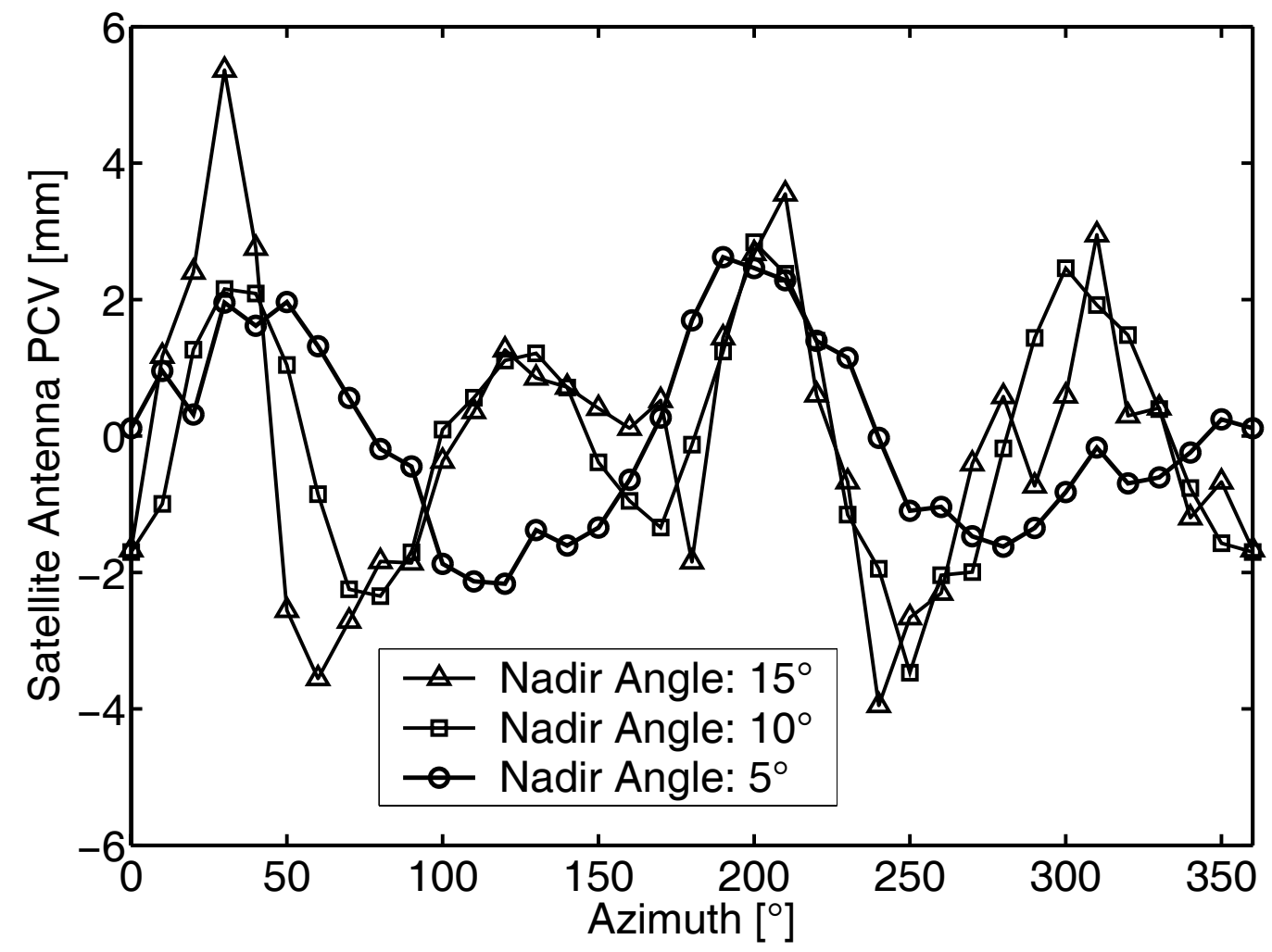

Fig. 11

Mean azimuthal satellite antenna PCVs of Block II/IIA for different nadir angles (July 14-19, 2002)

\section{Impact on global GPS solutions}

Besides demonstrating a new systematic effect, it is important to clarify the question whether and how the estimation of global GPS parameters is affected. In this context, the effect of a possible transition from relative to absolute PCVs on site coordinates (e.g. on the global scale) as well as on tropospheric parameters is analyzed here. One set of daily, shortly called "relative", solutions was estimated using the official IGS PCV set igs_01.pcv that contains relative receiver antenna PCVs only. In contrast to that, the IGS test set pcv_abs_proposed11.tst containing absolute receiver and satellite antenna PCVs was introduced to compute the "absolute" solutions. The latter file only includes nadir-dependent satellite antenna PCVs, so that the azimuth dependence mentioned above is not taken into account here. For all the following investigations the data set from the second half of October 2002 was used.

First of all, Figure 12 gives the change in site coordinates caused by a transition from relative to absolute PCVs in the form of a histogram: number of stations per coordinate difference. The values shown for the north, east and height component are the result of a two-week combination. As different realizations of the geocenter caused a systematic effect of several mm, mainly in the east component, translations between the two coordinate sets were removed. The systematic 
change in height of about $8 \mathrm{~mm}$ (corresponding to about $1.2 \mathrm{ppb}$ ) is caused by the adoption of the ITRF2000 scale when deriving absolute satellite antenna PCVs, whereas the scale of the "relative" solutions was not consistent with the ITRF2000 scale. All things considered, one has to expect jumps of up to $5 \mathrm{~mm}$ in the horizontal position and up to $1 \mathrm{~cm}$ in the height component. However, 2-3 mm of these jumps are not caused by switching from relative to absolute phase center corrections, but by the fact that azimuth-dependent receiver antenna PCVs are taken into account. Such azimuth dependencies are only contained in pcv_abs_proposed11.tst, but not in igs_01.pcv.
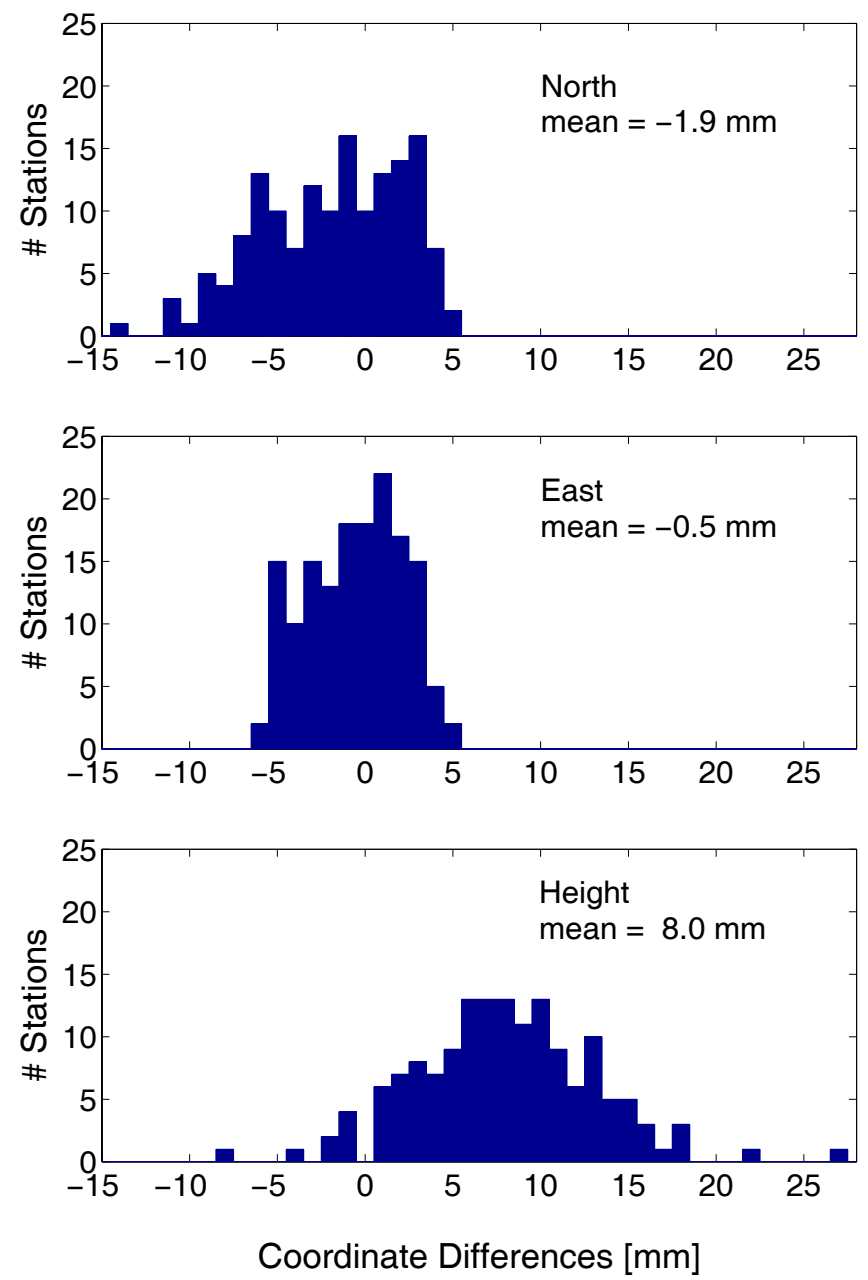

Fig. 12

Change in site coordinates when switching from relative to absolute PCVs (October 17-31, 2002; geocenter differences removed)

Several parameters, namely station heights, tropospheric delays, clock biases and (receiver as well as satellite) antenna phase center corrections are highly correlated. If the modeling of any of these parameters fails for low-elevation observations, coordinate results usually depend on the selected elevation cut-off angle (e.g. Elósegui et al. 1995, Hatanaka et al. 2001). In the case of relative 
receiver antenna PCVs, the behavior of the phase center is even unknown for low elevations, as the calibration in the field does not permit the estimation of PCVs below an elevation angle of about $10^{\circ}$ (Mader 1999). For this reason, one has to extrapolate values when using the file igs_01.pcv. In contrast, this would not be necessary, if absolute PCVs stemming from robot calibrations were applied. As the robot is capable of tilting the antenna to be calibrated, PCVs can be estimated down to $0^{\circ}$ elevation or even below. Figure 13 shows the changes in height due to a decrease of the elevation cut-off angle from $10^{\circ}$ to $3^{\circ}$ for October 17,2002 . The missing phase center information in the case of relative PCVs is reflected in a much bigger variance of the height differences, compared to the use of absolute PCVs. We may add that, comparing cut-off angles of $15^{\circ}$ and $10^{\circ}$ (not shown here), the variance is almost the same with both sets of PCVs. But whereas in the case of relative PCVs the height change averages about $4 \mathrm{~mm}$ (corresponding to about 0.6 $\mathrm{ppb}$ ), the average change is reduced to about $-1 \mathrm{~mm}$, when absolute receiver and satellite antenna PCVs are applied. This last comparison shows the effect of the erroneous assumption that the PCVs of the reference antenna AOAD/M_T as well as of all satellite antennas are zero.
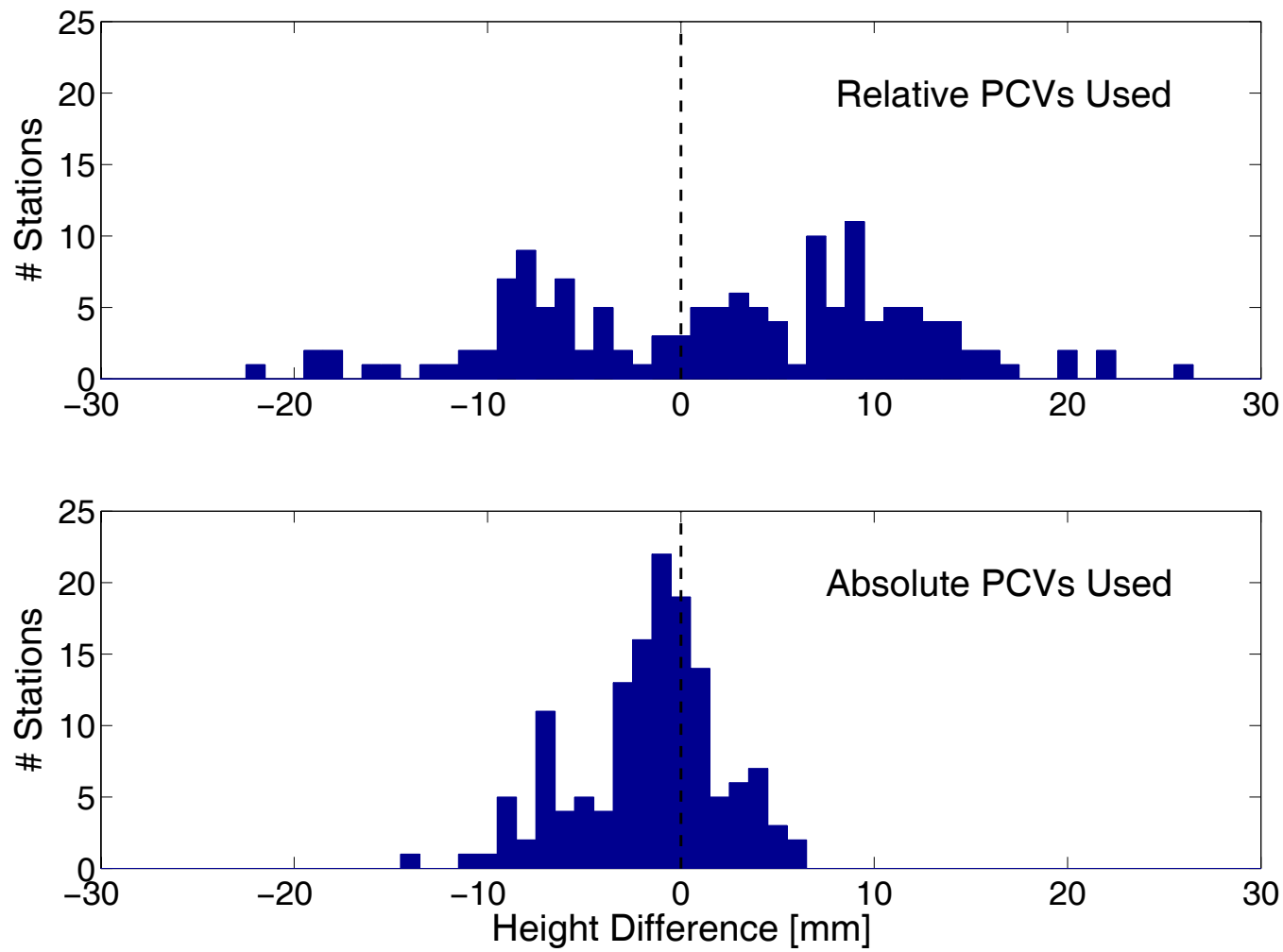

Fig. 13

Change in height due to a decrease of the elevation cut-off angle from $10^{\circ}$ to $3^{\circ}$ while using relative resp. absolute PCVs (October 17, 2002)

Besides the investigations of the coordinate results, also the tropospheric parameters are of particular interest, as they allow a comparison with independent space geodetic techniques, namely VLBI and DORIS, and with WVR observations as well. All signals traveling through the same 
atmosphere, each of these techniques should deliver equal estimates for the tropospheric zenith delay, except for a correction for the height difference between the individual instruments. As a rule of thumb, a height difference of $10 \mathrm{~m}$ between the GPS antenna and the VLBI telescope (or the WVR) causes a bias of approximately $3 \mathrm{~mm}$ in the tropospheric zenith delay (Saastamoinen 1973, see Table 1). This “theoretical bias" (a maximum of $7.5 \mathrm{~mm}$ for Algonquin in the network processed) that was computed from meteorological measurements is already accounted for in Figure 14 and Table 2. In both, the GPS and the VLBI analysis, a standard atmosphere was used to get Saastamoinen-based a priori $d r y$ zenith delays (cf. Hugentobler et al. 2001, p. 188) that were mapped with the dry Niell mapping function. For each interval of two hours one constant tropospheric delay per station (wet delay plus correction for the a priori dry part) was estimated, using the wet Niell mapping function. Besides, daily gradients in North and East direction were determined.

Table 1

Bias of the tropospheric zenith delay (dry and wet part) due to a height difference of $10 \mathrm{~m}$ between two instruments for different station heights (Saastamoinen 1973; standard atmosphere)

\begin{tabular}{|l|c|c|c|c|c|}
\hline Station height $h[\mathrm{~m}]$ & 0 & 500 & 1000 & 1500 & 2000 \\
\hline Tropospheric bias $\Delta \delta \rho_{\text {trop }}[\mathrm{mm}]$ & 3.9 & 3.3 & 2.9 & 2.6 & 2.4 \\
corresp. to $\Delta h=10 \mathrm{~m}$ & & & & & \\
\hline
\end{tabular}

The remaining biases in the tropospheric zenith delays between GPS and VLBI for eight colocated fundamental stations are given in Figure 14. Using relative PCVs, the bias is always positive and reaches values of up to $11.2 \mathrm{~mm}$ (Kokee Park), far beyond the accuracy of the individual techniques. The average bias improves from $5.4 \mathrm{~mm}$ to $-1.8 \mathrm{~mm}$ (see Table 2 ), if absolute PCVs are applied instead. The maximum bias of $-5.6 \mathrm{~mm}$ is obtained for Onsala then. It is noticeable that all three stations, whose bias is getting bigger by switching from relative to absolute PCVs (Fairbanks, Ny-Alesund and Onsala) are located at higher latitudes. At first consideration, this might lead to the conclusion that the tropospheric models do not match reality in these regions. However, the fact that exactly the GPS antennas at these three sites are equipped with radomes seems to be a more obvious explanation. For reasons of long-term consistency, calibration results for antenna/radome combinations have not been introduced within the IGS so far. The remaining biases between GPS and VLBI could have various reasons: elevationdependent and temperature- or gravity-induced deformations of the VLBI telescope, multipath effects or errors in the troposphere mapping functions. In Table 2 also the comparison of the GPS tropospheric results (wet part only) with a preliminary data set for one of the water vapor radiometers at Onsala is given. Taking into account the whole time span of two weeks, a bias of $4.9 \mathrm{~mm}$ results with relative, compared to only $-0.9 \mathrm{~mm}$ with absolute PCVs. 


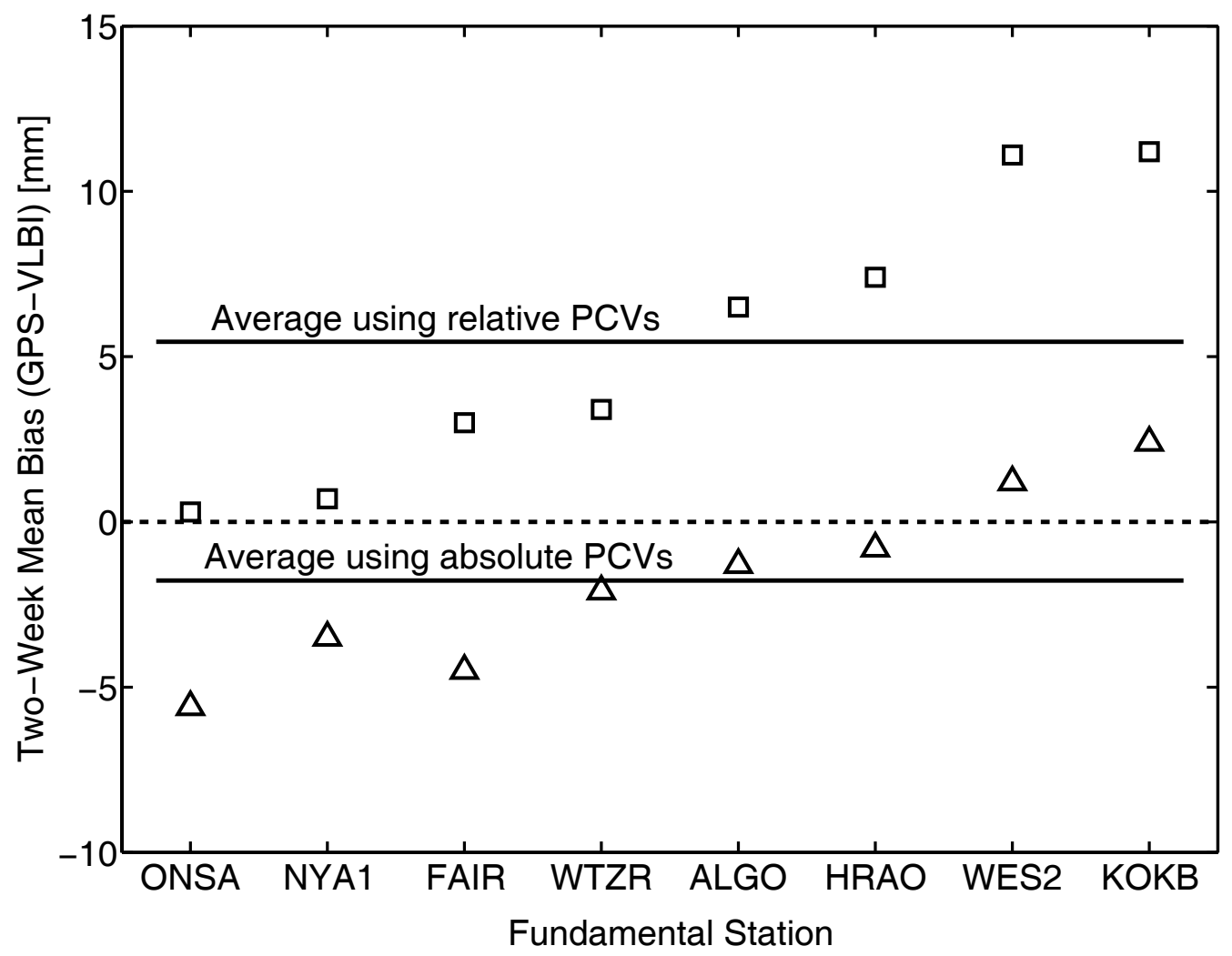

Fig. 14

Bias in the estimated tropospheric zenith delays between GPS and VLBI for eight co-located fundamental stations (October 17-31, 2002; height difference between the two instruments removed)

Table 2

Bias in the estimated tropospheric zenith delays between GPS, VLBI and WVR (October 17-31, 2002; height difference between the GPS antenna and the VLBI telescope removed)

\begin{tabular}{|c|c|c|c|c|}
\hline & $\begin{array}{c}\text { GPS - VLBI } \\
\text { (Mean of 8 } \\
\text { stations) }\end{array}$ & $\begin{array}{c}\text { GPS - VLBI } \\
\text { (Onsala) }\end{array}$ & $\begin{array}{c}\text { GPS - WVR } \\
\text { (Onsala, wet } \\
\text { part only) }\end{array}$ & $\begin{array}{c}\text { VLBI - WVR } \\
\text { (Onsala, wet } \\
\text { part only) }\end{array}$ \\
\hline relative PCVs & $\begin{array}{c}5.4 \mathrm{~mm} \\
\pm 4.3 \mathrm{~mm}\end{array}$ & $0.3 \mathrm{~mm}$ & $4.9 \mathrm{~mm}$ & \multirow{2}{*}{$3.5 \mathrm{~mm}$} \\
\hline absolute PCVs & $\begin{array}{c}-1.8 \mathrm{~mm} \\
\pm 2.7 \mathrm{~mm}\end{array}$ & $-5.6 \mathrm{~mm}$ & $-0.9 \mathrm{~mm}$ & \\
\hline
\end{tabular}

The tropospheric biases between GPS and VLBI resulting from the use of relative PCVs correspond well to the results of Schuh and Böhm (2003) who analyzed eleven co-located sites altogether. Their investigations also showed that the bias is always positive, the individual values ranging from $+1.4 \mathrm{~mm}$ to $+13.5 \mathrm{~mm}$ (Onsala: $+4.8 \mathrm{~mm}$ ). Haas et al. (2003) compared GPS and VLBI with data from water vapor radiometers and radiosondes, but for the Onsala Space 
Observatory only. The biases of the integrated precipitable water vapor (IPWV) they got from an analysis of long time series have to be multiplied by a factor of about 6 in order to get the biases of the wet zenith delay (Bouma 2002). From pairwise comparisons they got a bias of about $+2 \mathrm{~mm}$ and $+3.5 \mathrm{~mm}$ between GPS and VLBI and between GPS and WVR, respectively. A combination of the four techniques yielded biases of about $+0.5 \mathrm{~mm}$ (GPS - VLBI) and $+3 \mathrm{~mm}$ (GPS - WVR), however.

\section{Conclusions}

It has been shown that it is possible to estimate azimuth-dependent antenna PCVs of the GPS satellites from global GPS measurements. Although the results coincide very well with the expectations arising from the geometrical arrangement of the helical elements, it has to be annotated that still not enough is known about the satellite antennas. As the PCVs reach an order of magnitude of \pm 3-4 mm, they cannot be ignored in high-precision applications. The repeatability of the azimuthal PCVs between different satellites and from day to day as well as after several years is excellent. This leads to the conclusion that changes in the nadir-dependent PCVs over years might have a different cause, e.g. variations in the global scale.

The investigations of the coordinate results obtained with relative resp. absolute PCVs have shown that GPS antennas are a very critical error source. A transition from relative to absolute PCVs would cause jumps of 2-10 mm in all three components. The advantage would be, however, that the dependence of the coordinate results on the elevation cut-off angle could be significantly reduced. As the determination of relative PCVs is not possible down to the horizon, observations below an elevation angle of $10^{\circ}$ should not be used without switching to absolute PCVs.

The comparison of tropospheric parameters from GPS, VLBI and WVR has pointed out significant biases that complicate any effort in combining the different space geodetic techniques. It could be demonstrated that these biases are reduced significantly when switching from relative to absolute PCVs: an important step towards more consistency between the techniques. An inter-technique combination will only be beneficial, if each single technique corrects for its specific systematic effects. Another issue in this context could be highlighted once more: the problem that calibration results have to be available for any combination of antenna and radome.

The authors thank Dr. V. Tesmer (DGFI) for the analysis of the VLBI data and Prof. Dr. G. Elgered (Onsala Space Observatory) and his group for making available the preliminary WVR data set. 
Aparicio M, Brodie P, Doyle L, Rajan J, Torrione P (1995) GPS satellite and payload. In: Parkinson BW, Spilker JJ (eds) Global positioning system: theory and applications, vol. I. American Institute of Aeronautics and Astronautics, Inc., Washington, pp 209-244

Bouma HR (2002) Ground-based GPS in climate research. Technical report no. 456L, licentiate thesis at the School of Electrical and Computer Engineering, Chalmers University of Technology, Göteborg

Czopek FM, Shollenberger S (1993) Description and performance of the GPS Block I and II L-Band antenna and link budget. In: Proceedings of ION GPS-93, The Institute of Navigation, Salt Lake City, Utah, pp 37-43

Degnan JJ, Pavlis EC (1994) Laser ranging to GPS satellites with centimeter accuracy. GPS World, September 1994, pp 62-70

Elgered G, Haas R (2003) The geodetic VLBI network station at the Onsala Space Observatory - Activities during 2002. In: Schwegmann W, Thorandt V (eds) Proceedings of the 16th Working Meeting on European VLBI for Geodesy and Astrometry, Bundesamt für Kartographie und Geodäsie, Frankfurt/Leipzig, pp 61-66 Elósegui P, Davis JL, Jaldehag RTK, Johansson JM, Niell AE, Shapiro II (1995) Geodesy using the Global Positioning System: The effects of signal scattering on estimates of site position. Journal of Geophysical Research 100(B6): 9921-9934

Ge M, Gendt G (2005) Estimation and validation of the IGS absolute antenna phase center variations. In: Meindl M (ed) Proceedings of the IGS Workshop and Symposium 2004, to be published

Haas R, Elgered G, Gradinarsky L, Johansson J (2003) Assessing long term trends in the atmospheric water vapor content by combining data from VLBI, GPS, radiosondes and microwave radiometry. In: Schwegmann W, Thorandt V (eds) Proceedings of the 16th Working Meeting on European VLBI for Geodesy and Astrometry, Bundesamt für Kartographie und Geodäsie, Frankfurt/Leipzig, pp 279-288

Haines B, Bar-Sever Y, Bertiger W, Desai S, Willis P (2004) One-centimeter orbit determination for Jason-1: New GPS-based strategies. Marine Geodesy 27(1-2): 299-318. DOI 10.1080/01490410490465300

Hatanaka Y, Sawada M, Horita A, Kusaka M, Johnson JM, Rocken C (2001) Calibration of antenna-radome and monument-multipath effect of GEONET - Part 2: Evaluation of the phase map by GEONET data. Earth Planets Space 53(1): 23-30

Hugentobler U, Schaer S, Fridez P (2001) Bernese GPS Software, version 4.2. Astronomical Institute, University of Bern

Hugentobler U, Dach R, Fridez P (2005) Bernese GPS Software, version 5.0. Astronomical Institute, University of Bern, to be published

Mader GL (1999) GPS antenna calibration at the National Geodetic Survey. GPS Solutions 3(1): 50-58

Mader GL, Czopek FM (2002) The Block IIA satellite - Calibrating antenna phase centers. GPS World 13(5): 40-46

Rothacher M (2001) Comparison of absolute and relative antenna phase center variations. GPS Solutions 4(4): 55-60

Rummel R, Drewes H, Bosch W, Hornik H (2000) Towards an Integrated Global Geodetic Observing System (IGGOS). International Association of Geodesy Symposia, Vol. 120, Springer-Verlag, Heidelberg, pp 1-261

Rummel R, Drewes H, Beutler G (2002) Integrated Global Geodetic Observing System (IGGOS): A candidate IAG project. In: Adam J, Schwarz K-P (eds) Vistas for Geodesy in the New Millenium. International Association of Geodesy Symposia, Vol. 125, Springer-Verlag, New York, pp 609-614 Saastamoinen J (1973) Contribution to the theory of atmospheric refraction. Bulletin Géodésique 107: 13-34 
Schmid R, Rothacher M (2003) Estimation of elevation-dependent satellite antenna phase center variations of GPS satellites. Journal of Geodesy 77(7-8): 440-446. DOI 10.1007/s00190-003-0339-0

Schmid R, Mader GL, Herring TA (2005) From relative to absolute antenna phase center corrections. In: Meindl M (ed) Proceedings of the IGS Workshop and Symposium 2004, to be published

Schuh H, Böhm J (2003) Determination of tropospheric parameters within the new IVS Pilot Project. In: Schwegmann W, Thorandt V (eds) Proceedings of the 16th Working Meeting on European VLBI for Geodesy and Astrometry, Bundesamt für Kartographie und Geodäsie, Frankfurt/Leipzig, pp 257-264

Steigenberger P, Rothacher M, Dietrich R, Rülke A, Fritsche M (2004) Reprocessing of a global GPS network. Geophysical Research Abstracts, Vol. 6, 07649, European Geosciences Union

Thaller D, Schmid R, Rothacher M, Tesmer V, Angermann D, (2005) Rigorous combination of VLBI and GPS using the CONT02 campaign. In: IAG-Proceedings of the IUGG XXIII General Assembly, Sapporo, Japan, to be published

Titov O, Tesmer V, Böhm J (2001) OCCAM 5.0 users guide, AUSLIG Technical Report 7, AUSLIG, Canberra, Australia

Wübbena G, Schmitz M, Menge F, Böder V, Seeber G (2000) Automated absolute field calibration of GPS antennas in real-time. In: Proc. of the ION GPS Meeting 2000, Salt Lake City, USA 